\title{
Effects of Monolaurin on Oral Microbe-Host Transcriptome and Metabolome
}

\author{
Viviam de Oliveira Silva ${ }^{1,2 \dagger}$, Luciano José Pereira ${ }^{3}$, Silvana Pasetto ${ }^{2}$, \\ Maike Paulino da Silva ${ }^{2}$, Jered Cope Meyers ${ }^{4}$ and Ramiro Mendonça Murata ${ }^{4,5 *}$
}

1 Department of Veterinary Medicine, Federal University of Lavras, Lavras, Brazil, ${ }^{2}$ Division of Periodontology, Diagnostic Sciences, Dental Hygiene and Biomedical Science, Herman Ostrow School of Dentistry, University of Southern California, Los Angeles, CA, United States, ${ }^{3}$ Department of Health Sciences, Federal University of Lavras, Lavras, Brazil, ${ }^{4}$ Department Foundational Sciences, School of Dental Medicine, East Carolina University, Greenville, NC, United States, ${ }^{5}$ Department of Microbiology and Immunology, Brody School of Medicine, East Carolina University, Greenville, NC, United States

The aim of this in vitro study was to evaluate the effects of monolaurin against Aggregatibacter actinomycetemcomitans ( $\mathrm{Aa}$ ) and determine their effects on the host transcriptome and metabolome, using an oral cell/bacteria co-culture dual-chamber model to mimic the human periodontium. For this, the Aa, was applied to cross the monolayer of epithelial keratinocytes (OBA-9) to reach the fibroblasts layer (HGF-1) in the basal chamber. The Monolaurin treatments ( 25 or $50 \mu \mathrm{M})$ were added immediately after the inoculation of the dual-chamber with $\mathrm{Aa}$. After $24 \mathrm{~h}$, the transcriptional factors and metabolites produced were quantified in the remaining cell layers (insert and basal chamber) and in supernatant released from the cells. The genes IL-1 $\alpha, I L-6, I L-18$, and TNF analyzed in HGF-1 concentrations showed a decreased expression when treated with both concentration of Monolaurin. In keratinocytes, the genes IL-6, IL18, and TNF presented a higher expression and the expression of $\mathrm{IL}-1 \alpha$ decreased when treated with the two cited concentrations. The production of glycerol and pyruvic acid increased, and the 2-deoxytetronic acid NIST, 4-aminobutyric acid, pinitol and glyceric acid, presented lower concentrations because of the treatment with 25 and/or $50 \mu \mathrm{M}$ of Monolaurin. Use of monolaurin modulated the immune response and metabolite production when administered for $24 \mathrm{~h}$ in a dual-chamber model inoculated with $A$. actinomycetemcomitans. In summary, this study indicates that monolaurin had antimicrobial activity and modulated the host immune response and metabolite production when administered for $24 \mathrm{~h}$ in a dual-chamber model inoculated with A. actinomycetemcomitans.

Keywords: Aggregatibacter actinomycetemcomitans, periodontal disease, immune system, fibroblast, keratinocyte, monolaurin

\section{INTRODUCTION}

Periodontal disease is characterized by bacterial infection associated with the presence of biofilm, resulting in chronic inflammation of the tooth supporting tissues leading to a progressive destruction of periodontal tissue and alveolar bone. Signs and symptoms of periodontitis can include gingival swelling, bleeding during brushing, periodontal pockets, increased spacing 
between the teeth and tooth loss (Irfan et al., 2001; Michaud et al., 2007; Savage et al., 2009; Gurav, 2014).

The most common form of periodontal disease, denominated periodontitis, affects approximately $50 \%$ of the adult population with its severe forms affecting $10-15 \%$ of these individuals (Chapple and Genco, 2013).

The oral biofilm is composed of at least 800 different species of bacteria (Paster et al., 2006; Filoche et al., 2010). Numerous periodontopathogenic bacteria (Porphyromonas gingivalis, Fusobacterium nucleatum, Streptococcus intermedius, and others) are responsible for the initiation and maintenance of periodontal inflammation (Coussens and Werb, 2002; Oringer, 2002; Loos, 2005; Moutsopoulos and Madianos, 2006; de Almeida et al., 2007). The Aggregatibacter actinomycetemcomitans is a fermentative, gram-negative and capnophilic coccobacillus. It is not only considered the most important agent of localized aggressive periodontitis lesions, but is associated with chronic periodontitis as well (Slots et al., 1980; Zambon et al., 1983; Haraszthy et al., 2000; Gafan et al., 2004; Yang et al., 2004; Cortelli et al., 2009; Rakić et al., 2010; da Silva-Boghossian et al., 2011). The progression of periodontal disease is associated with the virulence factors of the microorganism, together with the vulnerability of the host. Aa produces leukotoxin, adhesins, bacteriocins, and lipopolysaccharide, which are responsible for interacting with the host cells triggering an inflammatory response in the periodontium (Cortelli et al., 2009). In addition, another critical factor for soft tissue inflammation and bone resorption is the immuneinflammatory host response to the bacterial biofilm (Salvi and Lang, 2005).

According to Aimetti (2014), non-surgical periodontal treatments possess limitations, such as the long-term maintainability of deep periodontal pockets, risk of disease recurrence, and skill of the operator. The development of new therapeutic agents that have the ability to inhibit the biofilm formation and modulate the inflammatory response can have a major impact in the prevention and treatment of periodontal disease. Monolaurin, also known as glycerol monolaurate or lauroyl, is a natural surfactant compound commonly used in cosmetics and the food industry. It is recognized by the Food and Drug Administration (FDA) as a GRAS (Generally Recognized as Safe) food additive since 1977. This natural compound is found in coconut oil and human breast milk (Fu et al., 2006; Peterson and Schlievert, 2006).

Monolaurin presents antibacterial and antiviral activity in vitro (Projan et al., 1994; Isaacs, 2001; Preuss et al., 2005; Peterson and Schlievert, 2006; Carpo et al., 2007), which may be of great interest in the treatment and/or prevention of various infections. However, the effect of monolaurin on periodontopathogenic bacteria has yet to be determined.

Therefore, the aims of this in vitro study were to evaluate the cytotoxicity, the antimicrobial effect of monolaurin against A. actinomycetemcomitans and to determine its effect on the host transcriptome and metabolome using a gum cell - bacteria co-culture model.

\section{MATERIALS AND METHODS}

\section{Monolaurin}

For carrying out the experiments, the monolaurin utilized was 1-lauroyl-rac-glycerol (Sigma-Aldrich, St. Louis, MO, United States). For dilution, sterilized deionized water was used as the vehicle.

\section{Cells and Bacterial Strain}

Human gingival fibroblast - HGF-1 (ATCC ${ }^{\circledR}$ CRL-2014) were cultured in Dulbecco's Modified Eagle's Medium (DMEM) (Lonza, Walkersville, MD, United States) with 10\% Fetal Bovine Serum (FBS) (Lonza, Walkersville, MD, United States). Human gingival epithelial cells (keratinocyte OBA-9) were cultured in specific medium for keratinocytes, Defined Keratinocyte-SFM (Life Technologies, Carlsbad, CA, United States) (Oda and Watson, 1990; Kusumoto et al., 2004).

Aggregatibacter actinomycetemcomitans (strain D7S-1) was cultivated from a subgingival plaque from an AfricanAmerican female patient diagnosed with generalized aggressive periodontitis (Chen et al., 2010). The bacteria were grown in Trypticase Soy Broth (TSB) (Becton Dickinson, Franklin Lakes, NJ, United States).

\section{Antimicrobial Activity}

Monolaurin antimicrobial activity was evaluated in A. actinomycetemcomitans after $24 \mathrm{~h}$ of treatment. Microorganisms were inoculated $\left[1 \times 10^{6}\right.$ colony-forming unit per milliliter $(\mathrm{CFU} / \mathrm{mL})$ in a 96-well microtiter plate with TSB (Becton Dickinson, Franklin Lakes, NJ, United States)] and monolaurin was immediately added in various concentrations $(0.5-1,000 \mu \mathrm{M})$ to determine the minimum inhibitory concentration (MIC) (Branco-de-Almeida et al., 2011). Microplates were maintained in a humidified incubator at $37^{\circ} \mathrm{C}$ and $5 \% \mathrm{CO}_{2}$. After incubation, bacterial growth was assayed by measurement of absorbance at $660 \mathrm{~nm}$. MIC was defined as the lowest concentration of monolaurin that had restricted growth to a level, 0.05 at $660 \mathrm{~nm}$ (no visible growth).

\section{Cytotoxicity Assay}

In vitro cytotoxicity effect was measured by the fluorometric resazurin method (O'Brien et al., 2000). OBA-9 or HGF-1 cells were seeded $\left(1 \times 10^{5}\right.$ cells $\left./ \mathrm{mL}\right)$ in 96-well microtiter plates and maintained in a humidified incubator at $37^{\circ} \mathrm{C}$ and $5 \% \mathrm{CO}_{2}$. After $24 \mathrm{~h}$, cell morphology was observed under an inverted microscope (EVOS FL Life Technologies, Carlsbad, CA, United States) to confirm that they had adhered to the bottom of each well and were presenting proper morphology. The monolaurin $(0.5-1,000 \mu \mathrm{M})$ was added to the cell cultures and incubated at $37^{\circ} \mathrm{C}$ and $5 \% \mathrm{CO}_{2}$. After $24 \mathrm{~h}$, the medium was discarded, cells were washed with room temperature phosphate buffered saline (PBS) (Lonza, Walkersville, MD, United States), and fresh, room temperature medium was added with resazurin (Cell Titer Blue Viability Assay - Promega Corp., Madison, WI, United States). Subsequently, the plate was incubated at $37^{\circ} \mathrm{C}$ and $5 \% \mathrm{CO}_{2}$. After $4 \mathrm{~h}$, the contents 
of the wells were transferred to a new microplate and the fluorescence was read in a microplate reader (SpectraMax M5, Molecular Devices, Sunnyvale, CA, United States) with excitation $550 \mathrm{~nm}$, emission $585 \mathrm{~nm}$, and cut off $570 \mathrm{~nm}$ (Pasetto et al., 2014). To calculate the results and obtain the $\mathrm{LD}_{50}$ (Lethal Dose), we performed a non-linear regression the program MasterPlex ReaderFit (Hitachi Solutions America).

\section{Dual-Chamber Model}

A dual-chamber, oral cell/bacteria co-culture was used to investigate the immunological effects of monolaurin (Silva et al., 2017). HGF- 1 cells $\left(1 \times 10^{5}\right)$ were seed in the basal chamber of a 24-well plate. Transwell inserts $\left(8 \mu \mathrm{m}\right.$ pore $\times 0.3 \mathrm{~cm}^{2}$ of culture surface - Greiner Bio-One, Monroe, NC, United States) were positioned in each well and OBA-9 cells $\left(1 \times 10^{5}\right)$ were seeded into the inserts. DMEM (Lonza, Walkersville, MD, United States) with $10 \%$ FBS (Lonza, Walkersville, MD, United States) was used for the medium. The plates were incubated at $37^{\circ} \mathrm{C}$ in humid air containing $5 \% \mathrm{CO}_{2}$ for $24 \mathrm{~h}$ (Silva et al., 2017). Trans Epithelial Electric Resistance (TEER) of each cell layer was measured with a Millicell-ERS Volt-Ohm Meter (Millipore, Bedford, MA, United States). Cell layer confluence in the Transwell insert was measured daily until reaching optimal TEER $\left(>150 \mathrm{Ohm} / \mathrm{cm}^{2}\right)$ after $48 \mathrm{~h}$. Once reaching optimal TEER, the media of the basal chamber and the insert were replaced with new media containing A. actinomycetemcomitans $\left(1 \times 10^{6} \mathrm{CFU} / \mathrm{mL}\right)$. Medium containing the microorganism was added to the insert, passing through the upper layer of cells (OBA-9) and reaching the bottom cell layer (HGF-1). Immediately after the inoculation of oral cell/bacteria co-culture with $A$. actinomycetemcomitans the monolaurin treatments (25 and $50 \mu \mathrm{M}$ ) were added and the plate was incubated at $37^{\circ} \mathrm{C}$ in humid air containing $5 \% \mathrm{CO}_{2}$. The time of exposure of the microorganism to monolaurin was $24 \mathrm{~h}$. Each experiment was repeated three times with two replicates per group $(n=6)$. The experiment was divided in three groups: (1) Control - oral cell/bacteria co-culture with $\mathrm{Aa}$ inoculated and no treatment; (2) Mono 25 - oral cell/bacteria co-culture with Aa inoculated and treated with $25 \mu \mathrm{M}$ of monolaurin; (3) Mono 50 - oral cell/bacteria co-culture with Aa inoculated and treated with $50 \mu \mathrm{M}$ of monolaurin.

\section{Collecting Samples for Analysis}

After the treatment period, liquid contents from each well was collected and centrifuged at 1,200 rpm for $10 \mathrm{~min}$. Following centrifugation, supernatant was divided into two aliquots for enzyme-linked immunosorbent assay (ELISA) and metabolome analysis. RNA was isolated from the remaining cell layers of HGF1 and OBA-9 (surface of the wells and inserts) for gene analysis in quantitative real-time PCR.

\section{ELISA Assay}

Inflammatory cytokines were determined using a commercial ELISA (Multi-Analyte ELISArray - Qiagen, Valencia, CA, United States). The cytokines were measured by standard ELISA protocol using a panel of 12 inflammatory cytokines: interleukin1 alpha (IL-1 $\alpha$ ), interleukin-1 beta (IL-1 $\beta$ ), interleukin-2 (IL2), interleukin-4 (IL-4), interleukin-6 (IL-6), interleukin-8 (IL8), interleukin-10 (IL-10), interleukin-12 (IL-12), interleukin-17 alpha (IL-17 $\alpha$ ), interferon gamma (IFN $\gamma$ ), tumor necrosis factor alpha $(\mathrm{TNF} \alpha)$, Granulocyte-Macrophage Colony Stimulating Factor (GM-CSF). Cytokines that presented the greatest change in concentration were selected for detailed analysis. These cytokines were analyzed using a standard ELISA protocol (Single Analyte ELISA Kit - Qiagen, Valencia, CA, United States).

\section{Gene Expression - Quantitative Real-Time PCR}

Total RNA was extracted through a commercial RNA purification kit (Mini Kit Qiagen RNeasy Protocol - Qiagen, Valencia, CA, United States) and the purity and quantity were measured in a NanoPhotometer P360 (Implen, Westlake Village, CA, United States). Total RNA was converted into singlestranded cDNA using a high-capacity reverse transcription kit (QuantiTec Reverse Transcription Kit - Qiagen, Valencia, CA, United States). An array for evaluation of gene expression of the inflammatory response was completed by quantitative real-time PCR (Prime PCR Pathway Plate/Acute Inflammation Response - Bio-Rad, Hercules, CA, United States) from the cDNA obtained. Based on these results, six genes/primers were selected for detailed study: interleukin 1 alpha (IL-1 $\alpha$ ), interleukin 6 (IL-6), interleukin 18 (IL-18), caspase 3 (CASP3), matrix metallopeptidase 1 (MMP-1), and tumor necrosis factor (TNF) (QuantiTect Primer Assay - Qiagen, Valencia, CA, United States). QuantiTect SYBR Green PCR Kits (Qiagen, Valencia, CA, United States) were used to determine the gene expression of the selected primers. The reaction product was quantified by relative quantification using Glyceraldehyde 3-phosphate dehydrogenase (GAPDH) as the reference gene. Data were analyzed as fold-change compared to control. The cycle threshold was calculated and interpreted using CFX Connect (Bio-Rad, Hercules, CA, United States) (Pfaffl, 2001).

\section{Metabolome Analysis}

Samples for metabolome analysis were treated as described by Silva et al. (2017) and analyzed at the West Coast Metabolomics Center (UC Davis Genome Center, Davis, CA, United States) by means of gas chromatography/mass spectrometry (Agilent 6890, Santa Clara, CA/Leco Pegasus IV, St. Joseph, MI, United States). Then, the metabolites were compared with the standard library for proper identification (Fiehn and Kind, 2007).

\section{Statistical Analysis}

Data were expressed as mean \pm standard deviation. The comparison between the groups was made through analysis of variance (ANOVA) and when a difference was determined, the Bonferroni test was applied. Data were analyzed using GraphPad Prism (version 5.01, GraphPad Software, San Diego, United States). The level of significance was set at $p<0.05$. 


\section{RESULTS}

\section{Antimicrobial Activity and Cytotoxicity Assay}

In order to determine antimicrobial activity of monolaurin, a range of $0.5-1,000 \mu \mathrm{M}$ was tested. Antibacterial activity was detected at 25-50 $\mu \mathrm{M}$. The minimum inhibitory concentration (MIC) was established between 25 and $50 \mu \mathrm{M}$. The cytotoxicity assays were conducted in HGF-1 and OBA-9 cells and the results are shown in Figure 1. For HGF-1 the $\mathrm{LD}_{50}$ was $146 \mu \mathrm{M}$ of monolaurin and for OBA-9 the $\mathrm{LD}_{50}$ was $69 \mu \mathrm{M}$ of monolaurin.

\section{ELISA}

Enzyme-linked immunosorbent assay (ELISA) results are presented in Figure 2. Both treatments (25 and $50 \mu \mathrm{M}$ ) of monolaurin increased the concentrations of GM-CSF and IL- $1 \alpha$. The concentrations of IL- 6 and IL-10 increased only with the $50 \mu \mathrm{M}$ treatment. On the other hand, IL-1 $\beta$ decreased as a function of monolaurin.

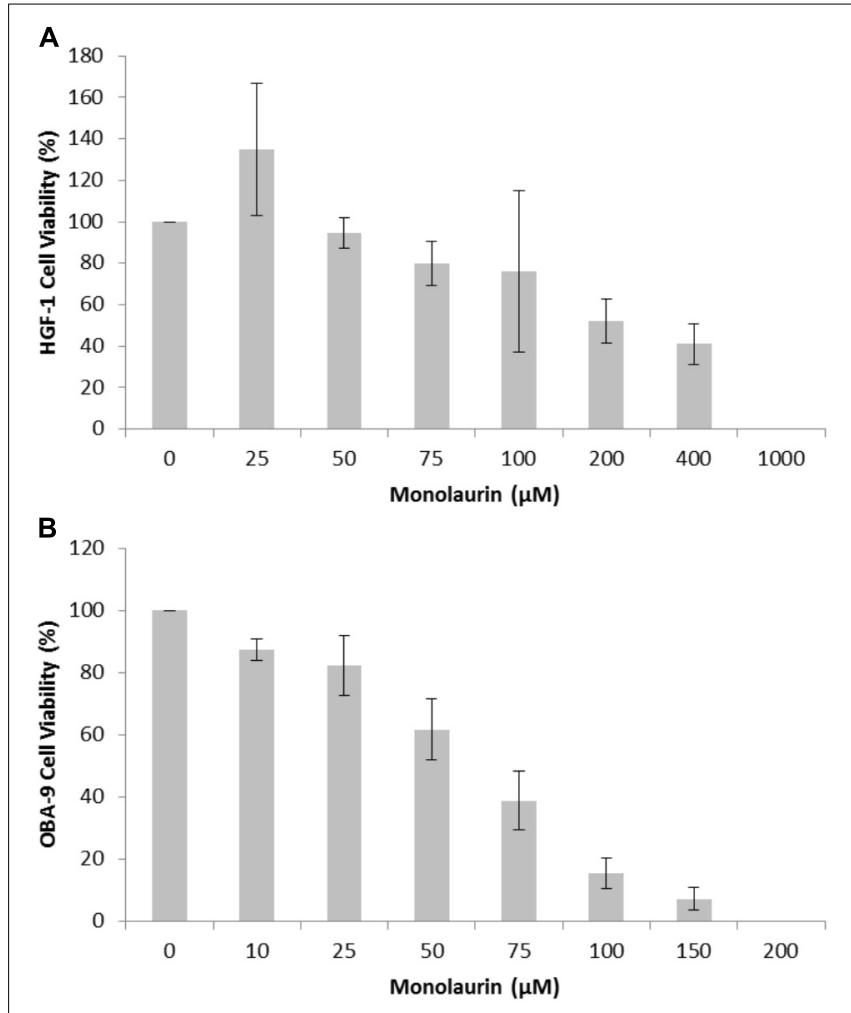

FIGURE 1 | Cytotoxicity assay of cells treated with different doses of monolaurin in an oral cell/bacteria co-culture inoculated with A. actinomycetemcomitans: (A) HGF-1 cell (fibroblasts); (B) OBA-9 cell (keratinocytes). Cell viability was presented in percentage $(\%) \pm$ standard deviation. Control group $=100 \%$ viability, $n=6$. Non-treated cells were considered as 100\% viability; DMSO was used as positive control to demonstrate an appropriate system-cell response (100\% cytotoxicity - data not shown).

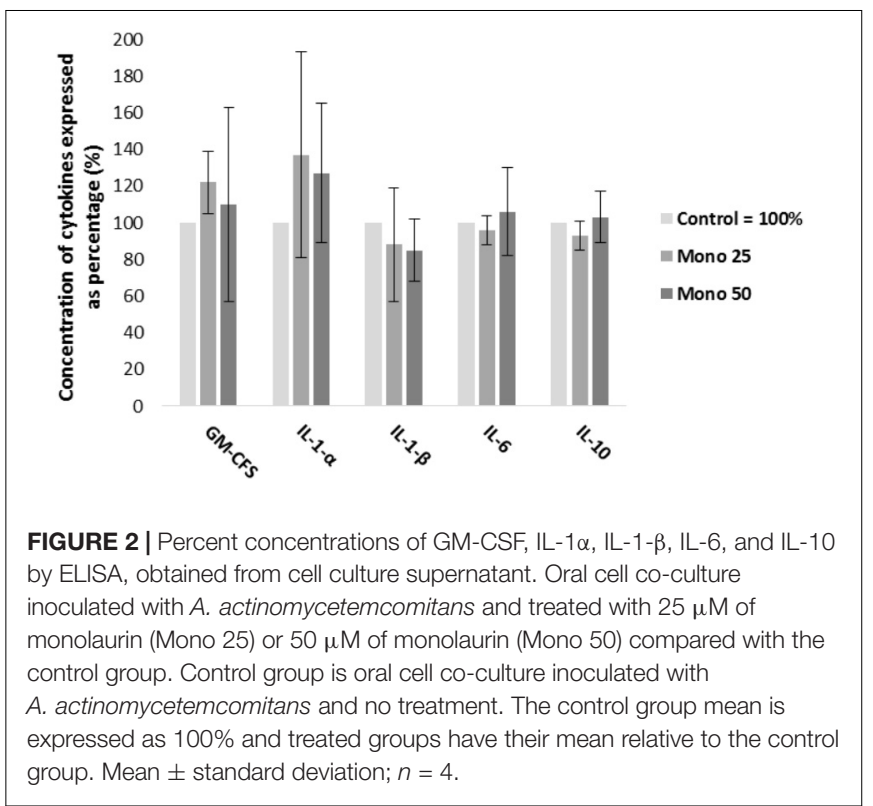

\section{Gene Expression - Quantitative Real-Time PCR}

Quantitative real-time PCR results are presented in Figures 3, 4. The genes IL- $1 \alpha$, IL- 6 , IL-18, and TNF analyzed in HGF- 1 showed a decreased expression $(p<0.05)$ when treated with 25 or $50 \mu \mathrm{M}$ of monolaurin compared with group Control (Figures 3A-C,F). CASP3 and MMP-1 genes showed no difference (Figures 3D,E).

Keratinocytes presented a higher IL-6, IL-18, and TNF gene expression $(p<0.05)$ when treated with 25 or $50 \mu \mathrm{M}$ of monolaurin compared with the control group (Figures $4 \mathbf{B}, \mathbf{C}, \mathbf{F}$ ). IL- $1 \alpha$ gene decreased expression $(p<0.05)$ in the group treated with both concentrations (Figure 4A). CASP-3 and MMP-1 genes showed no difference (Figures 4D,E).

\section{Metabolome}

The metabolome study returned a total of 283 metabolites. Of these, 120 were identified and only 6 presented a statistical difference as a function of monolaurin treatment. Some metabolites presented significantly altered concentrations (Figure 5). It was observed that both treatments of monolaurin increased the production of glycerol (Figure 5D) and pyruvic acid (Figure 5F) when compared to control. On the other hand, the 2-deoxytetronic acid NIST (Figure 5A) showed the opposite result, lower concentrations of this metabolite, in function of the treatment with 25 and $50 \mu \mathrm{M}$ of monolaurin. The $50 \mu \mathrm{M}$ monolaurin treatment group presented lower concentrations of 4-aminobutyric acid (Figure 5B), glyceric acid (Figure 5C), and pinitol (Figure 5E), against the control group.

\section{DISCUSSION}

Periodontal disease is known to be a widespread oral health problem across the world. Several methods for prevention and treatment of oral diseases have been used over time and new 

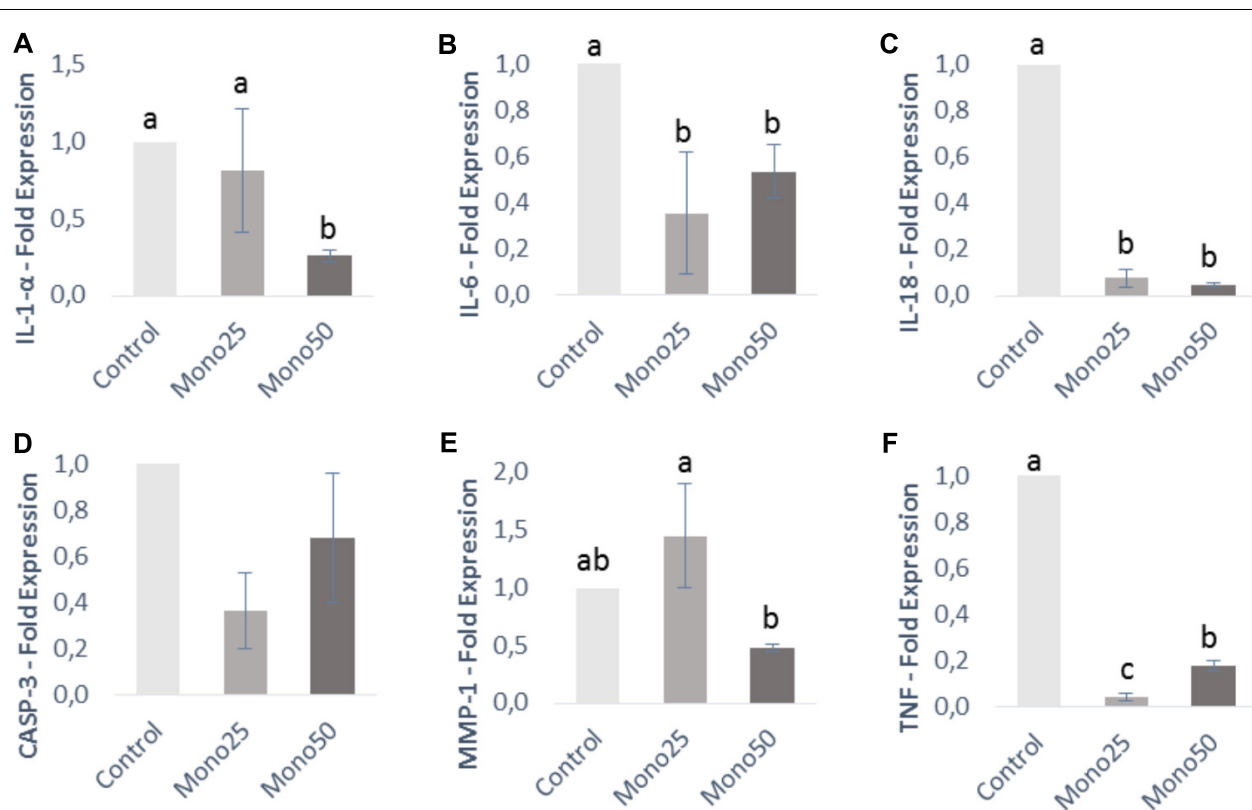

FIGURE 3 | Relative expression of genes from HGF-1 cells (fibroblasts) by quantitative PCR: (A) IL-1 $\alpha$; (B) IL-6; (C) IL-18; (D) CASP3; (E) MMP-1; (F) TNF. Oral cell/bacteria co-culture inoculated with A. actinomycetemcomitans and treated with different doses of monolaurin (25 $\mu \mathrm{M}-\mathrm{Mono} 25$ or $50 \mu \mathrm{M}-\mathrm{Mono} 50)$. Control group is oral cell co-culture inoculated with $A$. actinomycetemcomitans and no treatment. The control group has their mean expressed equal to 1 and treated groups have their mean relative to the control group. Different letters $(a, b$, and $c)$ indicate statistical difference between groups. The results were expressed by mean \pm standard deviation; $n=6$ and $p<0.05$.
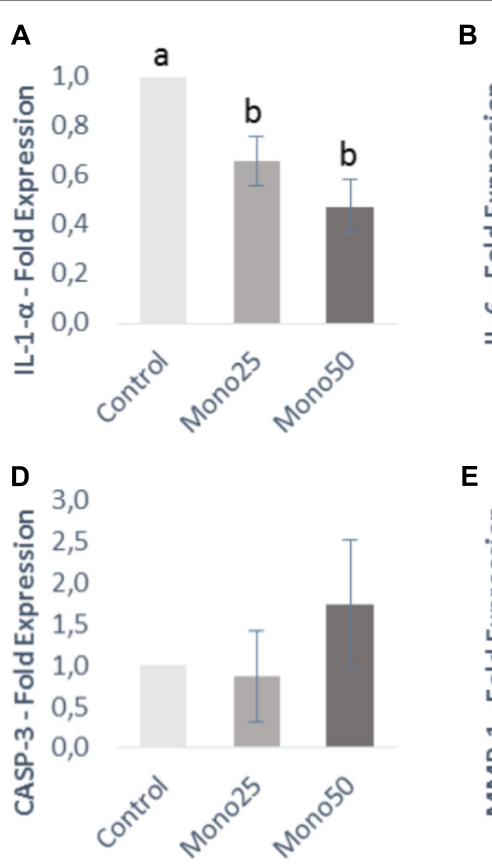

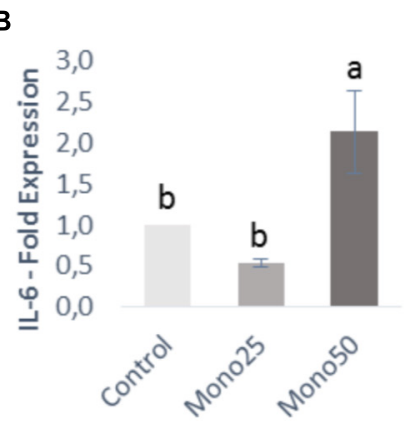

E

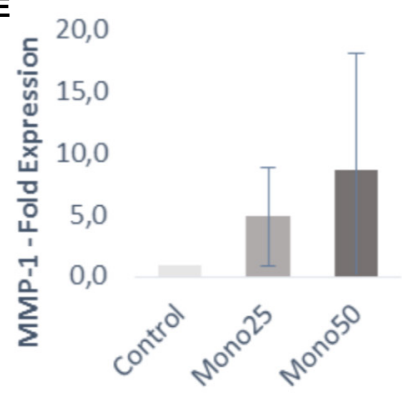

C

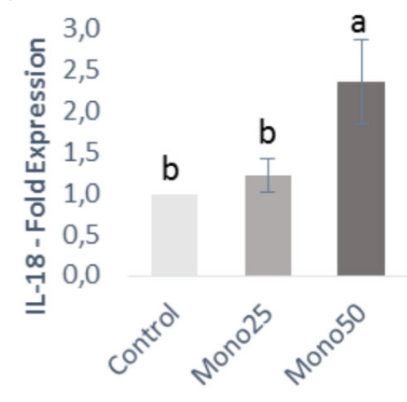

$\mathbf{F}$

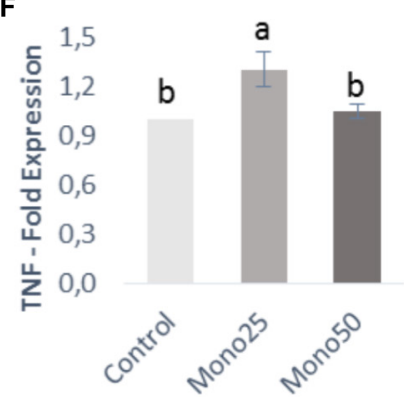

FIGURE 4 | Relative expression of genes from OBA-9 cells (keratinocytes) by quantitative PCR: (A) IL-1 $\alpha$; (B) IL-6; (C) IL-18; (D) CASP3; (E) MMP-1; (F) TNF. Oral cell/bacteria co-culture inoculated with $A$. actinomycetemcomitans and treated with different doses of monolaurin (25 $\mu \mathrm{M}-\mathrm{Mono} 25$ or $50 \mu \mathrm{M}-\mathrm{Mono} 50)$. Control group is oral cell co-culture inoculated with $A$. actinomycetemcomitans and no treatment. The control group has their mean expressed equal to 1 and treated groups have their mean relative to the control group. Different letters (a, b, and c) indicate statistical difference between groups. The results were expressed by mean \pm standard deviation; $n=6$ and $p<0.05$. 


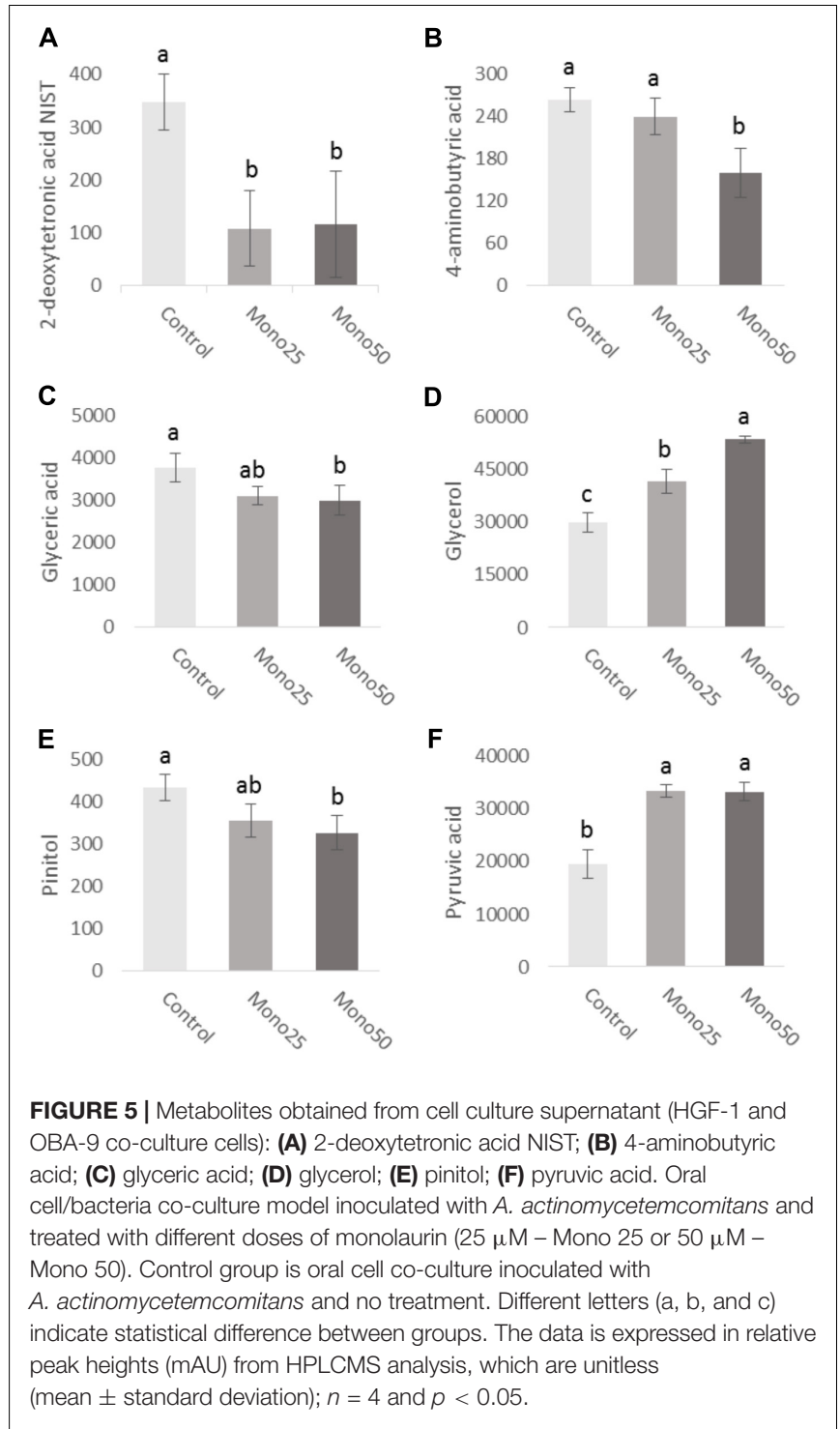

technologies are being developed. However, little attention has been given to the understanding of how periodontal structures are affected and how these structures react to different types of treatments (Balloni et al., 2016). Currently, some mouthwashes and toothpastes have been used as topical antimicrobial agents (Barnett, 2003). However, monolaurin is not listed as an active ingredient in commercially available mouthwashes. Monolaurin has been shown to possess diverse biological activities, such as antibacterial and antiviral properties (Preuss et al., 2005; Li et al., 2009; Tangwatcharin and Khopaibool, 2012; Seleem et al., 2016). In our in vitro study, susceptibility assays of monolaurin showed inhibition of microorganism grow at 25$50 \mu \mathrm{M}$ with minimal cytotoxicity. The putative pathways by which monolaurin affects Aa may involve: (i) increased membrane permeability and cell lysis, (ii) disruption of electron transport chain and uncoupling oxidative phosphorylation, and (iii) inhibition of membrane enzymatic activities and nutrient uptake (Yoon et al., 2018). In addition, co-culture supernatant were assessed for expression of host pro-inflammatory cytokines, gene expression of inflammatory cytokines and metabolomic profile in human cells infected with A. actinomycetemcomitans.

The gingival tissue comprises the oral epithelium and superficial underlying connective tissue. These tissues are sites of initiation for inflammatory processes and the first to be affected by biofilm (Balloni et al., 2016). Gingival epithelial cells represent a physical barrier against bacteria and are involved in processes of innate immunity (Jang et al., 2015). Keratinocytes and fibroblasts represent the major cell types present in gingival epithelial tissues. They interact directly with pathogenic microorganisms and are able to express a variety of cytokines and chemokines such as IL$1 \alpha$, IL-6, IL-8, TNF, among others (Bodet et al., 2007; Groeger and Meyle, 2015). In the present study, there was a decrease in the gene expression of IL- $1 \alpha$, IL-6, and IL-18 and TNF in HGF-1 and an increase in the expression of IL-6, IL-18, and TNF in OBA9 cells treated with monolaurin indicating a positive modulation in the inflammatory response of these cells.

Interleukin-1 alpha is a polypeptide that plays several roles in tissue homeostasis, immunity and has a proinflammatory nature. It is produced mainly by macrophages and monocytes, but also epithelial cells and fibroblasts (Champagne et al., 2003; Balloni et al., 2016). IL-1 $\alpha$ plays a critical role in protecting the body against invaders, such as bacteria and viruses, and is involved in extracellular matrix and bone metabolism (bone resorption, disintegration, and removal of bone tissue that is no longer needed). Therefore, IL- $1 \alpha$ is considered a marker of periodontal disease (Govindarajan et al., 2015; Groeger and Meyle, 2015).

Here, we observed a decrease in the expression of IL- $1 \alpha$ by HGF-1 and OBA- 9 cells in the monolaurin-treated groups which may be related to a beneficial inflammatory response since, elevated levels of IL- $1 \alpha$ and IL- $1 \beta$ in the gingival crevicular fluid are commonly found in patients with periodontal disease. This increased production of IL-1 is associated with a higher inflammatory response to the bacterial stimulus, resulting in more severe disease as well as an unfavorable response to treatments (Kornman et al., 1997; Engebretson et al., 1999; McGuire and Nunn, 1999; Shirodaria et al., 2000).

Interleukin-6 is associated with acute phase reactions and is usually secreted concomitantly with other proinflammatory cytokines. Some authors relate this cytokine to the destruction of periodontal tissue by favoring the accumulation of inflammatory cells and by activating and releasing inflammatory mediators that in turn accentuate the response to periodontal disease (de Lima Oliveira et al., 2012; Zhang et al., 2016).

The kinetics of production of IL-18 against the action of bacteria is rapid, according to its proinflammatory nature. It plays a central role in systemic and local inflammation (Biet et al., 2002). One study evaluated IL-18 levels in crevicular gingival fluid in four groups of patients: healthy periodontium, gingivitis, chronic periodontitis, and aggressive periodontitis. The results demonstrated that IL-18 concentration in the crevicular gingival fluid was low in the group with healthy periodontium and progressively increased in groups from gingivitis, to aggressive periodontitis, and finally chronic periodontitis. As the inflammation increased, there was a concomitant increase in IL-18 level (Nair et al., 2016). In the current study, treatment with 
monolaurin decreased the IL-18 gene expression in fibroblasts, however, an opposite result was observed in keratinocytes, where IL-18 expression was increased in the group treated with $50 \mu \mathrm{M}$ monolaurin.

Tumor necrosis factor plays a central role in periodontitis, being associated with the disease because of its ability to induce destruction of connective tissue and its effects on bone resorption through the activation of osteoclasts. Thus, TNF is one of the first proinflammatory cytokines produced in response to infection by pathogenic bacteria (Stashenko et al., 1987; de Lima Oliveira et al., 2012; Zhang et al., 2016). Here, the authors observed a reduction in the gene expression of TNF in monolaurin treated fibroblasts. On the other hand, in keratinocytes, TNF expression was higher in cells treated with $25 \mu \mathrm{M}$ of this compound. Animal models challenged with $P$. gingivalis were treated with antimicrobial drugs (chlorhexidine, minocycline, and doxycycline) in order to evaluate its effect on the inflammatory response. Minocycline induced higher levels of TNF while chlorhexidine reduced TNF levels, this result led the authors to believe that these agents modified the inflammatory response to $P$. gingivalis regardless of its antimicrobial effect, each in its own way (Houri-Haddad et al., 2008). Overall, monolaurin modulate the expression of inflammatory cytokines, suggesting it may have a modulatory role on the host pro-inflammatory response to help eradicate the bacterial infection. Similarly, previous studies suggested that monolaurin play a significant role on $\mathrm{T}$ cell functions and signaling by altering TCR-induced LAT, PLC- $\gamma$, and AKT cluster formation PI3K-AKT signaling axis, and calcium influx which ultimately decrease the cytokine production (Zhang et al., 2016).

The knowledge of the metabolic profile can contribute to understanding of the periodontal disease course as well as identify possible metabolites as biomarkers. This study evaluated the effects caused by monolaurin on the cellular metabolome and found significant variations. Relative quantification revealed lower levels of 2-deoxytetronic acid NIST, 4-aminobutyric acid, glyceric acid and pinitol, and higher levels of glycerol and pyruvic acid in cells treated with 25 or $50 \mu \mathrm{M}$ monolaurin. Analysis of fibroblast metabolome (HGF) treated with IL-1 $\beta$ in combination with titanium dioxide nanoparticles $\left(\mathrm{TiO}_{2} \mathrm{NPs}\right)$, demonstrated that IL-1 $\beta$ induction reduced concentrations of primary metabolites, especially those of urea cycle, polyamine, $\mathrm{S}$-adenosylmethionine and glutathione synthetic pathways. The addition of $\mathrm{TiO}_{2}$ NPs further increased these metabolic changes induced by IL-1 $\beta$. These findings may be useful for the future establishment of new metabolic markers and therapeutic strategy for gingival inflammations (Garcia-Contreras et al., 2015). One study compared the salivary metabolic profile of healthy patients and patients with periodontal disease. Changes in several classes of metabolites have been observed in individuals with periodontal

\section{REFERENCES}

Aimetti, M. (2014). Nonsurgical periodontal treatment. Int. J. Esthet. Dent. 9, 251-267.

Balloni, S., Locci, P., Lumare, A., and Marinucci, L. (2016). Cytotoxicity of three commercial mouthrinses on extracellular matrix metabolism and human disease. According to the authors, such changes reflected an increase in host-bacterial interactions in the diseased state (Barnes et al., 2011).

\section{CONCLUSION}

In summary, this study indicates that monolaurin possesses antimicrobial activity and modulates the host immune response and modulates production when administered for $24 \mathrm{~h}$ in a dual-chamber model inoculated with A. actinomycetemcomitans. These findings demonstrate that monolaurin could be considered a potential candidate for in vivo studies, which may translate into its potential clinical use to treat pathogenic microbe-host interactions.

\section{AUTHOR CONTRIBUTIONS}

VS conceived and designed the experiments, performed the experiments, analyzed the data, performed the statistical analysis, and wrote the paper. LP conceived and designed the experiments, contributed to reagents, materials, and analysis tools, analyzed and critically reviewed the data. MS and SP performed the experiments and analyzed the data. JM analyzed the data, and critically reviewed and interpreted the data. RM conceived and designed the experiments, contributed to reagents, materials, and analysis tools, performed the experiments, analyzed and critically reviewed the data. All authors contributed to manuscript revision, read, and approved the submitted version.

\section{ACKNOWLEDGMENTS}

The authors thank to CAPES (Coordination of Improvement of Higher Education Personnel), FAPEMIG (Research Sponsoring Agency of the State of Minas Gerais), and CNPq (National Counsel of Technological and Scientific Development) for support. Research reported in this publication was also supported by the NIH/NCCIH under award number AT006507. The content is solely the responsibility of the authors and does not represent the official views of the National Institutes of Health.

\section{SUPPLEMENTARY MATERIAL}

The Supplementary Material for this article can be found online at: https://www.frontiersin.org/articles/10.3389/fmicb. 2018.02638/full\#supplementary-material

gingival cell behaviour. Toxicol. Vitr. 34, 88-96. doi: 10.1016/j.tiv.2016. 03.015

Barnes, V. M., Ciancio, S. G., Shibly, O., Xu, T., Devizio, W., Trivedi, H. M., et al. (2011). Metabolomics reveals elevated macromolecular degradation in periodontal disease. J. Dent. Res. 90, 1293-1297. doi: 10.1177/ 0022034511416240 
Barnett, M. L. (2003). The role of therapeutic antimicrobial mouthrinses in clinical practice: control of supragingival plaque and gingivitis. J. Am. Dent. Assoc. 134, 699-704. doi: 10.14219/jada.archive.2003.0255

Biet, F., Locht, C., and Kremer, L. (2002). Immunoregulatory functions of interleukin 18 and its role in defense against bacterial pathogens. J. Mol. Med. 80, 147-162. doi: 10.1007/s00109-001-0307-1

Bodet, C., Andrian, E., Tanabe, S.-I., and Grenier, D. (2007). Actinobacillus actinomycetemcomitans lipopolysaccharide regulates matrix metalloproteinase, tissue inhibitors of matrix metalloproteinase, and plasminogen activator production by human gingival fibroblasts: a potential role in connective tissue destructio. J. Cell. Physiol. 212, 189-194. doi: 10.1002/jcp.21018

Branco-de-Almeida, L. S., Murata, R. M., Franco, E. M., dos Santos, M. H., de Alencar, S. M., Koo, H., et al. (2011). Effects of 7-epiclusianone on Streptococcus mutans and caries development in rats. Planta Med. 77, 40-45. doi: 10.1055/s0030-1250121

Carpo, B. G., Verallo-Rowell, V. M., and Kabara, J. (2007). Novel antibacterial activity of monolaurin compared with conventional antibiotics against organisms from skin infections: an in vitro study. J. Drugs Dermatol. 6, 991-998.

Champagne, C. M. E., Buchanan, W., Reddy, M. S., Preisser, J. S., Beck, J. D., and Offenbacher, S. (2003). Potential for gingival crevice fluid measures as predictors of risk for periodontal diseases. Periodontol. 2000 31, 167-180. doi: 10.1034/j.1600-0757.2003.03110.x

Chapple, I. L. C., and Genco, R. (2013). Diabetes and periodontal diseases: consensus report of the Joint EFP/AAP Workshop on Periodontitis and Systemic Diseases. J. Clin. Periodontol. 40(Suppl. 1), S106-S112. doi: 10.1111/ jcpe. 12077

Chen, C., Kittichotirat, W., Chen, W., Downey, J. S., Si, Y., and Bumgarner, R. (2010). Genome sequence of naturally competent Aggregatibacter actinomycetemcomitans serotype a strain D7S-1. J. Bacteriol. 192, 2643-2644. doi: 10.1128/JB.00157-10

Cortelli, S. C., Costa, F. O., Kawai, T., Aquino, D. R., Franco, G. C. N., Ohara, K., et al. (2009). Diminished treatment response of periodontally diseased patients infected with the JP2 clone of Aggregatibacter (Actinobacillus) actinomycetemcomitans. J. Clin. Microbiol. 47, 2018-2025. doi: 10.1128/JCM. 00338-09

Coussens, L. M., and Werb, Z. (2002). Inflammation and cancer. Nature 420, 860-867. doi: 10.1038 /nature 01322

da Silva-Boghossian, C. M., do Souto, R. M., Luiz, R. R., and Colombo, A. P. V. (2011). Association of red complex, A. actinomycetemcomitans and non-oral bacteria with periodontal diseases. Arch. Oral Biol. 56, 899-906. doi: 10.1016/j. archoralbio.2011.02.009

de Almeida, J. M., Theodoro, L. H., Bosco, A. F., Nagata, M. J. H., Oshiiwa, M., and Garcia, V. G. (2007). Influence of photodynamic therapy on the development of ligature-induced periodontitis in rats. J. Periodontol. 78, 566-575. doi: 10.1902/ jop.2007.060214

de Lima Oliveira, A. P., de Faveri, M., Gursky, L. C., Mestnik, M. J., Feres, M., Haffajee, A. D., et al. (2012). Effects of periodontal therapy on GCF cytokines in generalized aggressive periodontitis subjects. J. Clin. Periodontol. 39, 295-302. doi: 10.1111/j.1600-051X.2011.01817.x

Engebretson, S. P., Lamster, I. B., Herrera-Abreu, M., Celenti, R. S., Timms, J. M., Chaudhary, A. G., et al. (1999). The influence of interleukin gene polymorphism on expression of interleukin-1beta and tumor necrosis factoralpha in periodontal tissue and gingival crevicular fluid. J. Periodontol. 70, 567-573. doi: 10.1902/jop.1999.70.6.567

Fiehn, O., and Kind, T. (2007). Metabolite profiling in blood plasma. Methods Mol. Biol. 358, 3-17. doi: 10.1007/978-1-59745-244-1_1

Filoche, S., Wong, L., and Sissons, C. H. (2010). Oral biofilms: emerging concepts in microbial ecology. J. Dent. Res. 89, 8-18. doi: 10.1177/0022034509351812

$\mathrm{Fu}, \mathrm{X}$., Feng, F., and Huang, B. (2006). Physicochemical characterization and evaluation of a microemulsion system for antimicrobial activity of glycerol monolaurate. Int. J. Pharm. 321, 171-175. doi: 10.1016/j.ijpharm.2006.05.019

Gafan, G. P., Lucas, V. S., Roberts, G. J., Petrie, A., Wilson, M., and Spratt, D. A. (2004). Prevalence of periodontal pathogens in dental plaque of children. J. Clin. Microbiol. 42, 4141-4146. doi: 10.1128/JCM.42.9.4141-4146.2004

Garcia-Contreras, R., Sugimoto, M., Umemura, N., Kaneko, M., Hatakeyama, Y., Soga, T., et al. (2015). Alteration of metabolomic profiles by titanium dioxide nanoparticles in human gingivitis model. Biomaterials 57, 33-40. doi: 10.1016/ j.biomaterials.2015.03.059
Govindarajan, K., Muthukumar, S., and Rangarao, S. (2015). Relationship between interleukin $1 \alpha$ levels in the gingival crevicular fluid in health and in inflammatory periodontal disease and periodontal inflamed surface area: a correlative study. J. Indian Soc. Periodontol. 19, 618-623. doi: 10.4103/0972124X.162197

Groeger, S. E., and Meyle, J. (2015). Epithelial barrier and oral bacterial infection. Periodontol. 2000 69, 46-67. doi: 10.1111/prd.12094

Gurav, A. N. (2014). The association of periodontitis and metabolic syndrome. Dent. Res. J. 11, 1-10.

Haraszthy, V. I., Hariharan, G., Tinoco, E. M., Cortelli, J. R., Lally, E. T., Davis, E., et al. (2000). Evidence for the role of highly leukotoxic Actinobacillus actinomycetemcomitans in the pathogenesis of localized juvenile and other forms of early-onset periodontitis. J. Periodontol. 71, 912-922. doi: 10.1902/jop. 2000.71.6.912

Houri-Haddad, Y., Halabi, A., and Soskolne, W. A. (2008). Inflammatory response to chlorhexidine, minocycline $\mathrm{HCl}$ and doxycycline $\mathrm{HCl}$ in an in vivo mouse model. J. Clin. Periodontol. 35, 783-788. doi: 10.1111/j.1600-051X.2008. 01290.x

Irfan, U. M., Dawson, D. V., and Bissada, N. F. (2001). Epidemiology of periodontal disease: a review and clinical perspectives. J. Int. Acad. Periodontol. 3, 14-21.

Isaacs, C. E. (2001). The antimicrobial function of milk lipids. Adv. Nutr. Res. 10, 271-285.

Jang, D. H., Bhawal, U. K., Min, H.-K., Kang, H. K., Abiko, Y., and Min, B.M. (2015). A transcriptional roadmap to the senescence and differentiation of human oral keratinocytes. J. Gerontol. A Biol. Sci. Med. Sci. 70, 20-32. doi: $10.1093 /$ gerona/glt212

Kornman, K. S., Crane, A., Wang, H. Y., di Giovine, F. S., Newman, M. G., Pirk, F. W., et al. (1997). The interleukin-1 genotype as a severity factor in adult periodontal disease. J. Clin. Periodontol. 24, 72-77. doi: 10.1111/j.1600-051X. 1997.tb01187.x

Kusumoto, Y., Hirano, H., Saitoh, K., Yamada, S., Takedachi, M., Nozaki, T., et al. (2004). Human gingival epithelial cells produce chemotactic factors interleukin- 8 and monocyte chemoattractant protein-1 after stimulation with Porphyromonas gingivalis via toll-like receptor 2. J. Periodontol. 75, 370-379. doi: 10.1902/jop.2004.75.3.370

Li, Q., Estes, J. D., Schlievert, P. M., Duan, L., Brosnahan, A. J., and Southern, P. J. (2009). Glycerol monolaurate prevents mucosal SIV transmission. Nature 458, 1034-1038. doi: 10.1038/nature 07831

Loos, B. G. (2005). Systemic markers of inflammation in periodontitis. J. Periodontol. 76, 2106-2115. doi: 10.1902/jop.2005.76.11-S.2106

McGuire, M. K., and Nunn, M. E. (1999). Prognosis versus actual outcome. IV. The effectiveness of clinical parameters and IL-1 genotype in accurately predicting prognoses and tooth survival. J. Periodontol. 70, 49-56. doi: 10.1902/jop.1999. 70.1.49

Michaud, D. S., Joshipura, K., Giovannucci, E., and Fuchs, C. S. (2007). A prospective study of periodontal disease and pancreatic cancer in US male health professionals. J. Natl. Cancer Inst. 99, 171-175. doi: 10.1093/jnci/ djk021

Moutsopoulos, N. M., and Madianos, P. N. (2006). Low-grade inflammation in chronic infectious diseases: paradigm of periodontal infections. Ann. N. Y. Acad. Sci. 1088, 251-264. doi: 10.1196/annals. 1366.032

Nair, V., Bandyopadhyay, P., Kundu, D., and Das, S. (2016). Estimation of interleukin-18 in the gingival crevicular fluid and serum of Bengali population with periodontal health and disease. J. Indian Soc. Periodontol. 20, 260-264. doi: 10.4103/0972-124X.183098

O'Brien, J., Wilson, I., Orton, T., and Pognan, F. (2000). Investigation of the Alamar Blue (resazurin) fluorescent dye for the assessment of mammalian cell cytotoxicity. Eur. J. Biochem. 267, 5421-5426. doi: 10.1046/j.1432-1327.2000. 01606.x

Oda, D., and Watson, E. (1990). Human oral epithelial cell culture I. Improved conditions for reproducible culture in serum-free medium. In vitro cell. Dev. Biol. 26, 589-595. doi: 10.1007/BF02624208

Oringer, R. J. (2002). Modulation of the host response in periodontal therapy. J. Periodontol. 73, 460-470. doi: 10.1902/jop.2002.73.4.460

Pasetto, S., Pardi, V., and Murata, R. M. (2014). Anti-HIV-1 activity of flavonoid myricetin on HIV-1 infection in a dual-chamber in vitro model. PLoS One 9:e115323. doi: 10.1371/journal.pone.0115323 
Paster, B. J., Olsen, I., Aas, J. A., and Dewhirst, F. E. (2006). The breadth of bacterial diversity in the human periodontal pocket and other oral sites. Periodontol. 2000 2006, 80-87. doi: 10.1111/j.1600-0757.2006.00174.x

Peterson, M. L., and Schlievert, P. M. (2006). Glycerol monolaurate inhibits the effects of Gram-positive select agents on eukaryotic cells. Biochemistry 45, 2387-2397. doi: 10.1021/bi051992u

Pfaffl, M. W. (2001). A new mathematical model for relative quantification in real-time RT-PCR. Nucleic Acids Res. 29:e45. doi: 10.1093/nar/29.9.e45

Preuss, H. G., Echard, B., Enig, M., Brook, I., and Elliott, T. B. (2005). Minimum inhibitory concentrations of herbal essential oils and monolaurin for grampositive and gram-negative bacteria. Mol. Cell. Biochem. 272, 29-34. doi: 10. 1007/s11010-005-6604-1

Projan, S. J., Brown-Skrobot, S., Schlievert, P. M., Vandenesch, F., and Novick, R. P. (1994). Glycerol monolaurate inhibits the production of beta-lactamase, toxic shock toxin-1, and other staphylococcal exoproteins by interfering with signal transduction. J. Bacteriol. 176, 4204-4209. doi: 10.1128/jb.176.14.42044209.1994

Rakić, M., Zelić, K., Pavlica, D., Hadzimihajlović, M., Milasin, J., Milicić, B., et al. (2010). [Association between clinical parameters and the presence of Aggregatibacter actinomycetemcomitans and Porphyromonas gingivalis in patients with progressive periodontal lesions]. Vojnosanit. Pregl. 67, 898-902. doi: 10.2298/VSP1011898R

Salvi, G. E., and Lang, N. P. (2005). Host response modulation in the management of periodontal diseases. J. Clin. Periodontol. 32(Suppl. 6), 108-129. doi: 10.1111/ j.1600-051X.2005.00785.x

Savage, A., Eaton, K. A., Moles, D. R., and Needleman, I. (2009). A systematic review of definitions of periodontitis and methods that have been used to identify this disease. J. Clin. Periodontol. 36, 458-467. doi: 10.1111/j.1600-051X. 2009.01408.x

Seleem, D., Chen, E., Benso, B., Pardi, V., and Murata, R. M. (2016). In vitro evaluation of antifungal activity of monolaurin against Candida albicans biofilms. PeerJ 4:e2148. doi: 10.7717/peerj.2148

Shirodaria, S., Smith, J., McKay, I. J., Kennett, C. N., and Hughes, F. J. (2000). Polymorphisms in the IL-1A gene are correlated with levels of interleukin-1alpha protein in gingival crevicular fluid of teeth with severe periodontal disease. J. Dent. Res. 79, 1864-1869. doi: 10.1177/002203450007901 10801

Silva, V., de, O., Pereira, L. J., and Murata, R. M. (2017). Oral microbehost interactions: influence of $\beta$-glucans on gene expression of inflammatory cytokines and metabolome profile. BMC Microbiol. 17:53. doi: 10.1186/s12866017-0946-1
Slots, J., Reynolds, H. S., and Genco, R. J. (1980). Actinobacillus actinomycetemcomitans in human periodontal disease: a crosssectional microbiological investigation. Infect. Immun. 29, 1013-1020.

Stashenko, P., Dewhirst, F. E., Peros, W. J., Kent, R. L., and Ago, J. M. (1987). Synergistic interactions between interleukin 1, tumor necrosis factor, and lymphotoxin in bone resorption. J. Immunol. 138, 1464-1468.

Tangwatcharin, P., and Khopaibool, P. (2012). Activity of virgin coconut oil, lauric acid or monolaurin in combination with lactic acid against Staphylococcus aureus. Southeast Asian J. Trop. Med. Public Health 43, 969-985.

Yang, H. W., Asikainen, S., Doğan, B., Suda, R., and Lai, C. H. (2004). Relationship of Actinobacillus actinomycetemcomitans serotype $\mathrm{b}$ to aggressive periodontitis: frequency in pure cultured isolates. J. Periodontol. 75, 592-599. doi: 10.1902/ jop.2004.75.4.592

Yoon, B. K., Jackman, J. A., Valle-González, E. R., and Cho, N. J. (2018). Antibacterial free fatty acids and monoglycerides: biological activities, experimental testing, and therapeutic applications. Int. J. Mol. Sci. 8:E1114. doi: 10.3390/ijms19041114

Zambon, J. J., Christersson, L. A., and Slots, J. (1983). Actinobacillus actinomycetemcomitans in human periodontal disease. Prevalence in patient groups and distribution of biotypes and serotypes within families. J. Periodontol. 54, 707-711. doi: 10.1902/jop.1983.54.12.707

Zhang, M. S., Sandouk, A., and Houtman, J. C. (2016). Glycerol Monolaurate (GML) inhibits human $\mathrm{T}$ cell signaling and function by disrupting lipid dynamics. Sci. Rep. 26:30225. doi: 10.1038/srep30225

Zhang, Q., Chen, B., Zhu, D., and Yan, F. (2016). Biomarker levels in gingival crevicular fluid of subjects with different periodontal conditions: a crosssectional study. Arch. Oral Biol. 72, 92-98. doi: 10.1016/j.archoralbio.2016. 08.020

Conflict of Interest Statement: The authors declare that the research was conducted in the absence of any commercial or financial relationships that could be construed as a potential conflict of interest.

Copyright (c) 2018 Silva, Pereira, Pasetto, da Silva, Meyers and Murata. This is an open-access article distributed under the terms of the Creative Commons Attribution License (CC BY). The use, distribution or reproduction in other forums is permitted, provided the original author(s) and the copyright owner(s) are credited and that the original publication in this journal is cited, in accordance with accepted academic practice. No use, distribution or reproduction is permitted which does not comply with these terms. 